\title{
Research on Crash Recovery Strategy for Distribution Network Containing DG
}

\author{
Di Bai ${ }^{1,2}$, Tieyan Zhang ${ }^{2}$, Zheng Yang ${ }^{2}$ \\ ${ }^{1}$ Shenyang Agricultural University, \\ 2 Shenyang Institute of Engineering \\ * Corresponding author: hopebaidi@163.com
}

\begin{abstract}
In order to fully utilize $D G$ and improve system reliability, the author puts forward the rash recovery strategy for distribution network with DG. It adopts the heuristic method by taking $D G$ as the source point of research for distribution network power recovery. Based on simplifying the network through improving branch method with the consideration of purposes of network damage to minimum extent and switch action time, it conducts reconstruction after the crash. It optimizes the power supply recovery strategy according to the load transfer condition after the crash in order to minimize the damage to distribution network caused by blackout. It conducts example test at IEEE33 node and IEEE69 node to verify the validity of this scheme.
\end{abstract}

Keywords: distributed generation, power supply recovery, islanding classification, distribution network, branch switch method

\section{Introduction}

Currently, under the dual press coming from energy requirement and environmental protection, the distributed generation (DG) technologies that can improve the traditional energy's use rate and fully utilize various renewable powers are drawing more and more attentions. The use of DG can better utilize the clean and renewable energies in all places, provide "green energy" for users and realize "energy saving and emission reduction." [1]

As the effective supplementation of centralized generation, DG power generating technologies are becoming more and more mature and they are widely applied in distribution network. [2] The access of DG in large amount brings huge changes to the structure of distribution network and the increase of distributed generation puts forward new requirements for the power grid operation. [3] For example, when crash happens to the distribution network, the ways of operating DG are related to compiling crash recovery strategy. In order to fully utilize DG and elevate system reliability, IEEE released one new programmatic islanding standard IEEE1547, which encourages users to use islanding operation after crash happens to power generating enterprises and users, namely, conduct separation of power grid with DG from public power grid after the crash happens and DG will be responsible for independently supplying power for the isolated power grids. [4]

In this paper, it analyzes the DG distribution network's islanding operation method and puts forward one islanding classification method; namely, use the heuristic algorithm taking DG as the source point of research for distribution network power recovery. Based on simplifying the network model through improving branch method, it conducts reconstruction after the crash. Optimize the power recovery strategy in order to minimize the damage to distribution network caused by blackout. 


\section{DG Islanding Classification}

\subsection{Constrained Conditions for Islanding Classification}

Distribution network's islanding classification based on distribution network crash isolating scheme should meet with the operation conditions with the consideration of conditions as follows ${ }^{[5]}$ :

(1) Capacity constraint -- the sum between total amount of internal load and loss in island should not be larger than total power generation capacity of DG;

(2) Voltage constraint -- it should meet with requirement for node voltage;

(3) Power constraint -- branch power should be less than or equal to maximum permitted power;

(4) Connection constraint -- all nodes in the island should be connected with each other and they should be connected with DG.

\subsection{Confirm Power Loss Area}

Confirm power loss area based on the result of crash point and depth search number and the classification of island in power loss area will improve the classification efficiency:

(1) Power loss node array initiation UNN [0];

(2) Search for all nodes taking crash nodes as the father nodes and save them in power loss node array UNN [1];

(3) Search for all nodes taking internal nodes in power loss node array UNN[1] as the father nodes and save them in power loss node array UNN[2];

(4) Repeat step (3), update power loss node array UNN[i] until it is impossible to add new node, that is, UNN $[i+1]=\mathrm{UNN}[i]$;

It is noticeable that island should be adjacent to the distribution network terminal, that is, when you search to confirm the island range; you need to search first the downstream power loss areas in DG.

\subsection{Islanding Classification Algorithm}

(1) It adopts operation on DG by using adjacency matrix, that is, search for the next matrix unit in adjacency matrix for one DG. Assume that the next matrix unit meets with , the node load is extended to island to form the new DG unit and modify the unit adjacency matrix before extension according to the changes after unit extension. If the new DG is adjacent to other DG units, the 2 DG units will be merged into one new unit before we modify adjacency matrix according to the extension situation of the new unit;

(2) Repeat step (1) to realize the DG unit extension to maximum extent until the capacity of any DG unit matrix element is smaller than any load unit in adjacent matrix element.

(3) After Step (2) is finished, inspect all load units in unit adjacency matrix one by one. Once you detect one load Unit $S_{m}$ are adjacent to two or more than two DG Units $S_{D G p}$. $S_{D G q} S_{m}$, you need to extend them to new units before you modify unit adjacency maxtrix.

(4) Repeat Step (3) until it is impossible for extension. 
(5) Judge whether or not new DG units are generated in Step (3) and (4) and judge whether or not Step (1) is real. If it is real, return to Step (1) for execution; otherwise, the algorithm ends here. The following is the algorithm flow chart:

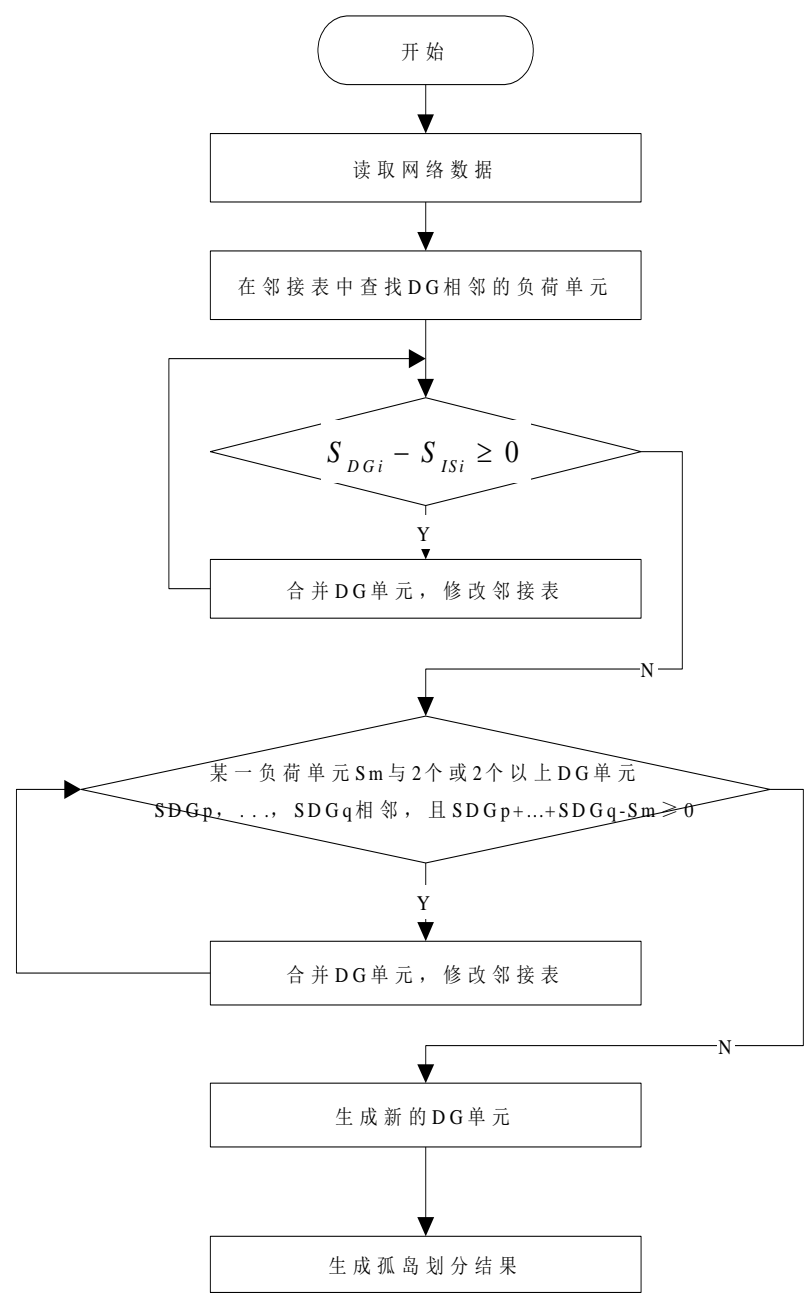

Figure 1. Islanding Classification Scheme Flow Chart

\section{Mathematical Model of Distribution Network Crash Recovery}

\subsection{Objective Function in Crash Recovery}

(1) Recover power loss load amount to maximum extent

$$
\max f_{1}=\sum_{i \in \mathrm{D}} \alpha_{i} L_{i}
$$

In the formula: $L_{\mathrm{i}}$ is power loss load; $\alpha_{\mathrm{i}}$ is weight coefficient of power loss load and it symbolizes load level; $\mathbf{D}$ is load aggregation when power supply is not restored;

(2) Switch operation time is at the minimum extent

$$
\max f_{2}=\sum_{\mathrm{k} \in \mathrm{S}}\left(1-\mathrm{K}_{\mathrm{k}}\right)+\sum_{\mathrm{k} \in \mathrm{T}} \mathrm{K}_{\mathrm{k}}
$$


In the formula: $\mathrm{T}$ is interconnection switch aggregation before the crash; $\mathrm{S}$ is segment switch aggregation before the crash; $R_{b}$ is switch status, 1 is OFF and 0 is $\mathrm{ON}$;

(3) Network damage to minimum extent

$$
\max f_{3}=\sum_{\mathrm{b}=1}^{\mathrm{n}} \mathrm{I}_{\mathrm{b}}^{2} \mathrm{R}_{\mathrm{b}}
$$

In the formula: $f_{3}$ is power loss in distribution network; $\mathrm{I}_{\mathrm{b}}$ is the effective value of current in branch $\mathrm{b} ; \mathrm{R}_{\mathrm{b}}$ is resistance in branch $\mathrm{b}$.

\subsection{Constraining Conditions for Crash Recovery}

(1) Network Tide Constraint

$$
\dot{U}_{i} \sum Y_{i j}^{*} \dot{U}_{j}^{*}=P_{i}+j Q_{i}
$$

In the formula: $\dot{U}_{i}$ and $\dot{U}_{j}$ are node voltages; $Y_{i j}$ is mutual admittance between nodes; $P_{i}+j Q_{i}$ is injected power in node;

(2) Limitation by Guarantee Voltage and Tide Stability

$$
\begin{gathered}
S_{i} \leq S_{i \cdot \max } \quad i \in B \\
V_{i, \min } \leq V_{i} \leq V_{i \cdot \max } \quad i \in N
\end{gathered}
$$

In the formula: $\mathrm{Si}$, max is power transmission limit in branch; $\mathrm{V} \mathrm{i}$, max are $\mathrm{V} \mathrm{i}$, min are respectively the upper limit and lower limit of node voltage;

(3) Limitation by System Radial Power Supply

$$
\begin{gathered}
n_{0}=m_{0} \\
n_{i}=m_{i}+1 \quad i=1,2, \ldots, N_{D}
\end{gathered}
$$

In the formula: $\mathrm{n} 0$ and $\mathrm{m} 0$ are respectively the number of nodes and number of branches in the residual system except the island; ni and mi are respectively the number of nodes and number of branches in No. $i$ island.

\section{Crash Recovery and Optimization of Residual Distribution Network}

\subsection{Residual Network Crash Recovery}

It adopts heuristic power supply recovery method. Arrange the order from large Figure to small Figure of the spare capacity of interconnection switches to form $t s$ list. If $t s$ list is blank, transfer load through secondary interconnection switch according to steps as follows:

(1) Calculate the transferable load $I_{\mathrm{ss}}$ of each section switch $s s$ with the largest spare capacity between $t s_{A}$ and $t s_{1}$; 
(2) Search for section switch ss with $I_{\mathrm{ss}}$ less than spare capacity $I_{\mathrm{M}}$ at maximum value, which is symbolized by $s s_{A}$; if all $I_{\mathrm{ss}}$ are larger than $I_{\mathrm{M}}$, it is unable to transfer load. In this circumstance, select the next interconnection switch in $t s$ list.

(3) If $t s_{A}$ exists, turn off $t s_{A}$ and turn on $s s_{A}$ and conduct tide verification of the feeder where $t s_{A}$ is located; if safety off-limit appears, enter Step (4); otherwise, enter Step (5);

(4) Select one ss combination at downstream of $t s_{A}$ and $s s_{A}$ as the candidate switch pair. Take it as the new section switch and return to Step (3). If there is no candidate section switch, delete interconnection switch $t s_{A}$ and select the next interconnection switch in $t s$ list and return to Step (1); repeat the acts until there is no safety off-limit or there is no $t s$;

(5) Select the $s s_{A}$ corresponding to $t s_{A}$, turn off $t s_{A}$ and turn on $s s_{A}$ and search ends.

Refer to Diagram 2 for Flow Chart.

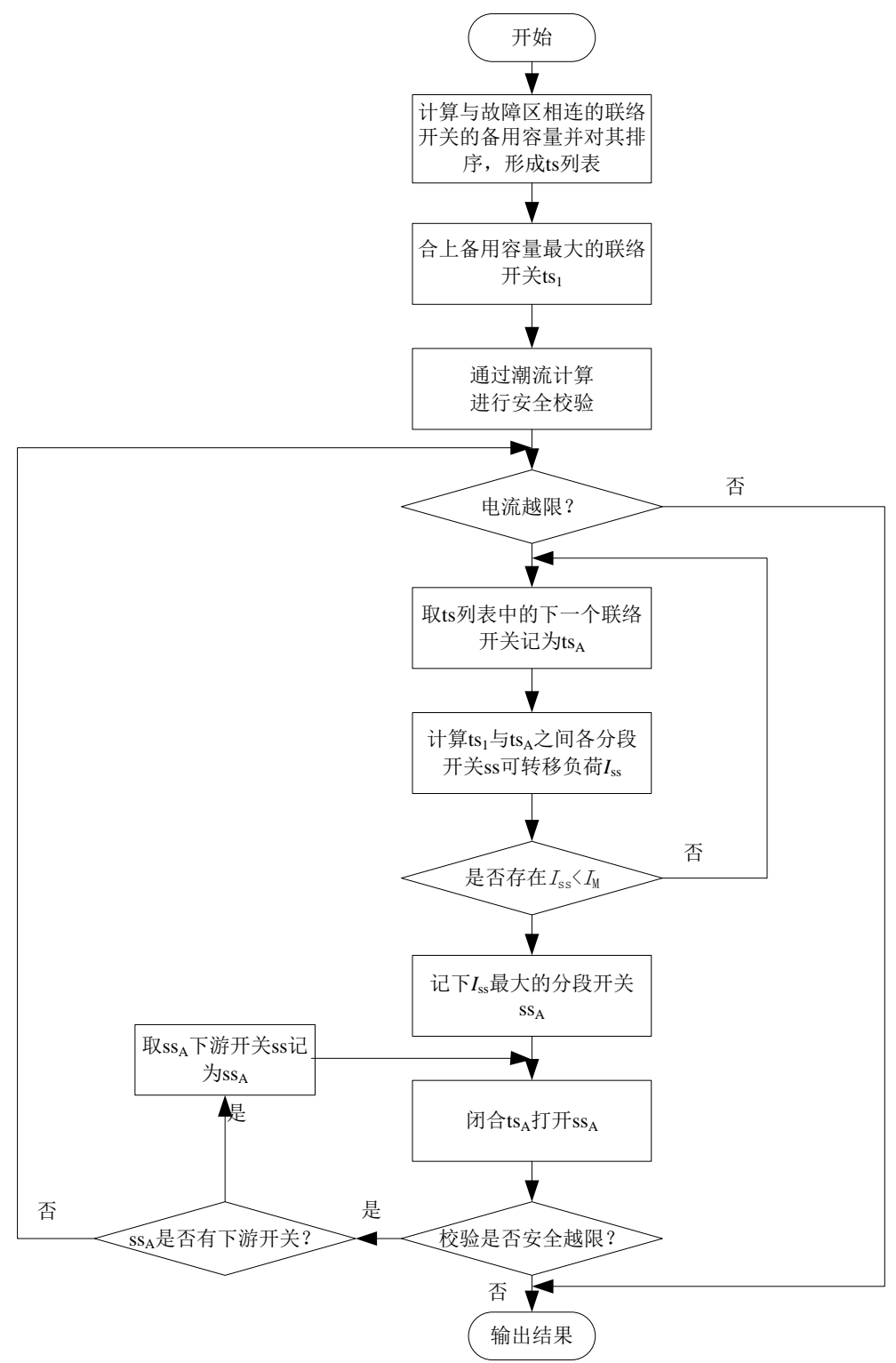

Figure 2. Flow Chart of Interconnection Switch's Power Supply Recovery 


\subsection{Optimization after Network Crash Recovery}

Optimization after Network Crash Recovery aims at realizing the minimum damage to network. As to the reconstruction for distribution network with DG island, it uses branch switch method, namely, after the loop is formed after one interconnection switch is turned off, you need to turn on the section switch that can realize the minimum damage to network damage; load will be transferred from node $\mathrm{n}$ to node $\mathrm{m}$ and the system network damage change formula is as follows:

$$
\Delta P=\operatorname{Re}\left\{2\left(\sum_{i \in D} I_{i}\right)\left(E_{m}-E_{n}\right)^{*}\right\}+R_{\text {loop }} \cdot\left|\sum_{i \in D} I_{i}\right|^{2}
$$

In the formula, $\sum_{i \in D} I_{i}$ is sum of transferred load; $R_{\text {loop }}$ is the sum of resistance in loop formed by turning off the interconnection switch;

We can obtain mother line voltage through $E_{t}=\sum_{j=1}^{x} R_{\text {bus }}(k, j) I_{j}$, in which $R_{b u s}$ is mother line resistance, $\mathrm{x}$ is the number of nodes, $\mathrm{t}=\mathrm{m}, \mathrm{n}$; We can see that if $\Delta P$ is negative, it indicates the reduction of active power loss; if $\Delta P$ positive, it indicates the increase of active power loss; We can improve the above-mentioned branch switch method and simplify the formula as follows:

$$
\Delta P=2 \times I_{x}\left(V_{m}-V_{n}\right)+R_{\text {loop }}\left|I_{x}\right|^{2}
$$

When we select the opened switch, we need to make current $I_{x}=\sum_{i \in D} I_{i}$ approach $I\left(x_{\text {opt }}\right)=\frac{E_{n}-E_{m}}{R_{\text {loop }}}$ as possible as we can; in this circumstance, $\Delta P_{\min }=\frac{\left(E_{n}-E_{m}\right)^{2}}{R_{\text {loop }}}$.

Based on this simple method, we can reduce network loss through confirming one switch change. Repeat searching for all switches and we will realize the option of reducing network damage to maximum extent until there is no network damage reduction.

Specific methods are as follows:

(1) Calculate distribution network tide before switch operation to obtain the voltage of all nodes and tides in all branch routes;

(2) When the voltage difference between the two ends reaches the maximum value and after turn off the switch, the load is transferred from node with low voltage to node with high voltage, turn off the next interconnection switch $t s_{A}$;

(3) If the left branch aggregation in the loop formed by interconnection switch $t s_{A}$ is $\boldsymbol{B} \boldsymbol{L}_{j}$ and right branch aggregation is $\boldsymbol{B} \boldsymbol{R}_{j}$, when section switch $s s_{k}, k \in B L_{j}$ or $k \in B R_{j}$ is turned on, the power on the interconnection switch is transferred as $S_{k}=P_{k}+j Q_{k}$ and the branch resistance is at $r_{k}$, then system network damage is as follows:

$$
P_{\text {Loss }}(j, k)=P_{\text {loop }}(j, k)+P_{o t}
$$




$$
P_{o t}=\left(P_{k}^{2}+Q_{k}^{2}\right) \cdot r_{k}
$$

In the formula, $P_{o t}$ is network damage in non-loop system; $P_{\text {loop }}$ is network damage in loop system; when node voltage approaches 1p.u, then

$$
\begin{gathered}
P_{\text {Loss }}(j, k)=P_{\text {loop }}^{L}(j, k)+P_{\text {loop }}^{R}(j, k) \\
P_{\text {loop }}^{L}(j, k)=\sum_{i \in B L_{j}}\left[\left(P_{i}-P_{k}\right)^{2}+\left(Q_{i}-Q_{k}\right)^{2}\right] \cdot r_{i} \\
P_{\text {loop }}^{R}(j, k)=\sum_{i \in B R}\left[\left(P_{i}+P_{k}\right)^{2}+\left(Q_{i}+Q_{k}\right)^{2}\right] \cdot r_{i}
\end{gathered}
$$

In order to realize the purpose of network damage to minimum extent, formula (11) needs to meet with following condition:

$$
\frac{\partial P_{\text {Loss }}(j, k)}{\partial P_{k}}=\frac{\partial P_{\text {Loss }}(j, k)}{\partial Q_{k}}=0
$$

Therefore, we can confirm the optimum amount of load transfer at:

$$
\begin{aligned}
P_{k}^{o p t}= & \frac{\sum_{i \in B L_{j}} P_{i} r_{i}-\sum_{i \in B R_{j}} P_{i} r_{i}}{\sum_{i \in B L_{j} \cup B R_{j}} r_{i}+r_{k}} \\
Q_{k}^{o p t}= & \frac{\sum_{i \in B L_{j}} Q_{i} r_{i}-\sum_{i \in B R_{j}} Q_{i} r_{i}}{\sum_{i \in B L_{j} \cup B R_{j}} r_{i}+r_{k}}
\end{aligned}
$$

We can calculate $P_{k}^{o p t}+Q_{k}^{o p t}$,that is, best transferrable load, and look for the branch power approaching $P_{k}^{\text {opt }}+Q_{k}^{\text {opt }}$ in branch aggregation; the branch switch and interconnection switch on this branch constitute the maximum damage reduction switch change in this loop network.

\section{Example Analysis}

\subsection{IEEE 33 Node System}

In IEEE33 node system, there are 37 branches and 5 interconnection switches with rated voltage at $12.66 \mathrm{kv}$ as illustrated in Diagram 1 and its system's specific parameters are extracted from document [6]. Install DG with installation capacity at $400 \mathrm{~kW}$ at Node 16 and Node 22. Assume the crash happens to position between branch 14 and 15 as illustrated in Fig. 3. 


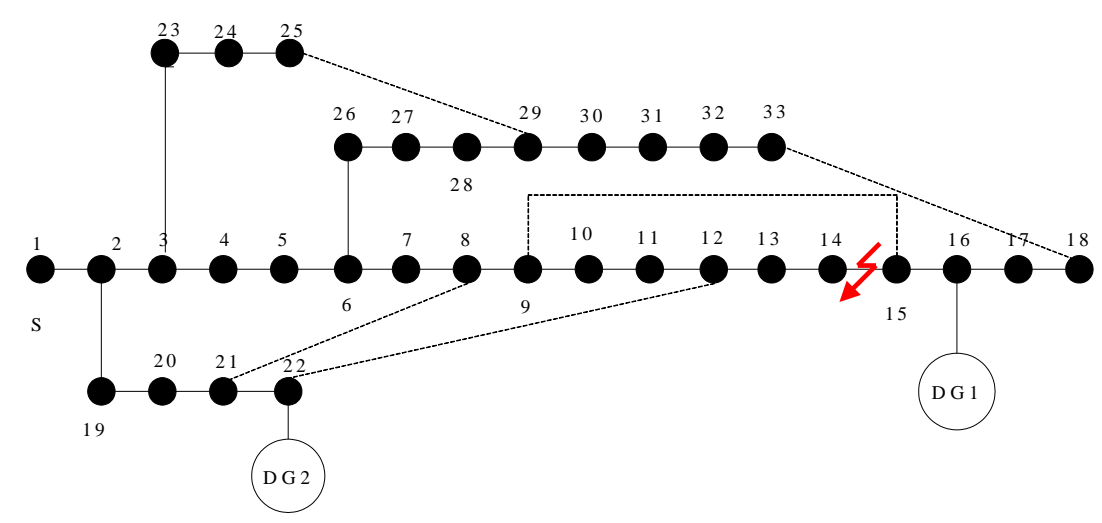

Figure 3. IEEE33 Node System Chart with Two DG

When DG fails to conduct emergent power generation, DG will be cut off when crash appears. Conduct crash recovery switch action according to Table 1 and the power loss load after recovery is 0 . Network loss before crash is $134.98 \mathrm{~kW}$ and network loss after recovery is $134.48 \mathrm{~kW}$ and the network loss is reduced by using this method.

When DG is able to conduct emergent power generation, DG will form islanding power supply after crash and network recovery result is as illustrated in Diagram 4. Total network loss including DG island is $63.59 \mathrm{kw}$, which indicates that network loss is largely reduced when it is able to guarantee the recovery of full power supply load.

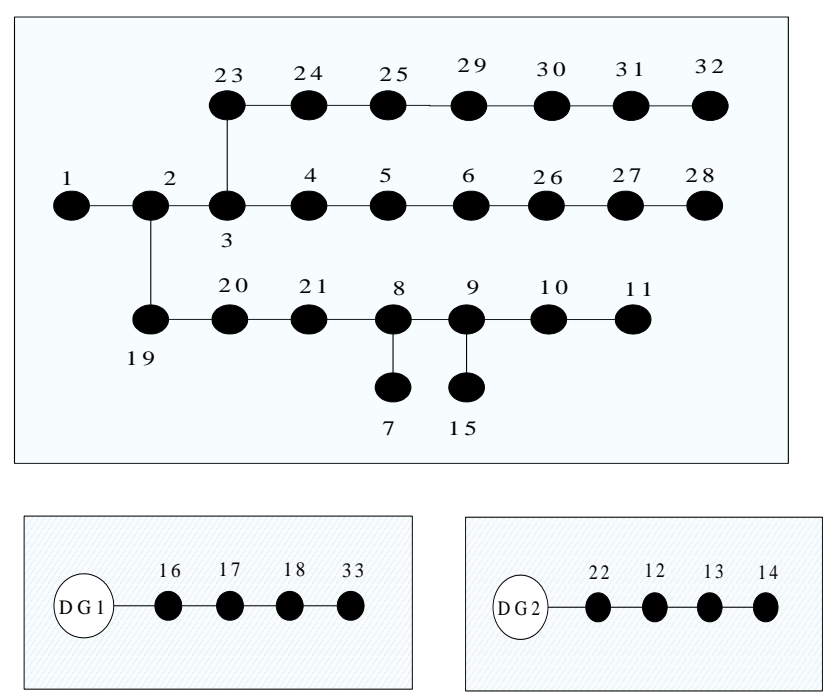

Figure 4. Reconstruction Structure of Distribution Network with Two DG

Table 1. Network Reconstruction Switch Action Result

\begin{tabular}{cc}
\hline $\begin{array}{c}\text { Network } \\
\text { Condition }\end{array}$ & Opened Switches \\
\hline $\begin{array}{c}\text { Excluding DG } \\
\text { Including DG }\end{array}$ & $8-9,14-15,10-11,32-33,29-30$ \\
& $6-7,28-29,21-22,11-12,14-15,15-16,32-33$, \\
\hline
\end{tabular}


Taking IEEE33 Node System as an example (refer to Diagram 5): respectively install DG on node 16, 22 and 28 for grid-connected operation with capacity at $350 \mathrm{~kW}$. Crash happens to place between Branch 14 and 15 as illustrated in Fig. 5.

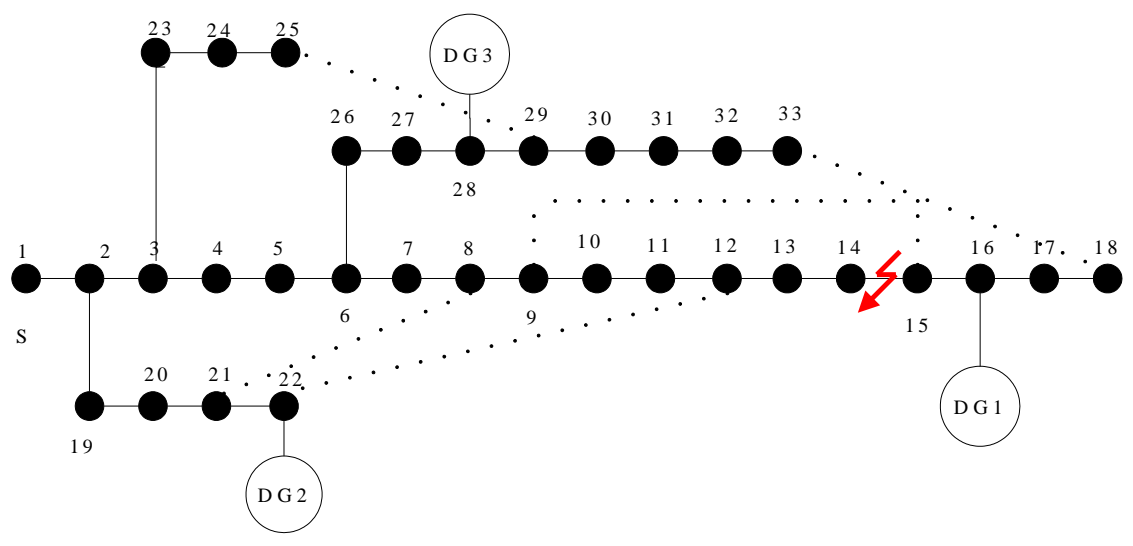

Figure 5. Diagram of leee33 Node System with Three DG

Using this method for calculation: when DG is able to conduct emergent power generation, the total network loss is $75.15 \mathrm{~kW}$. Islanding Scheme after crash is recovered is as illustrated as that in Fig. 6. Please refer to Table 2 for changes of switches.

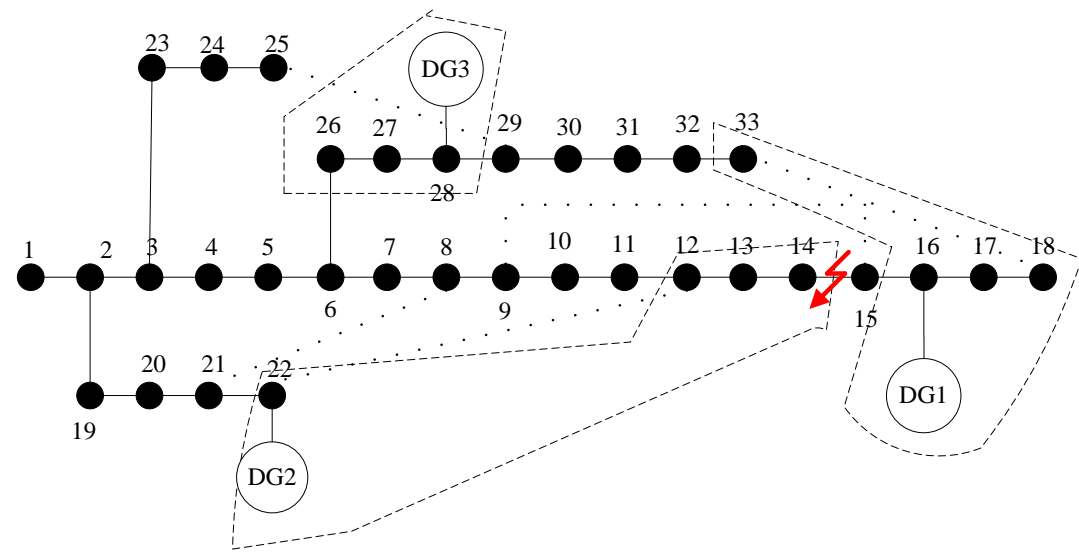

Figure 6. Reconfiguration Result of IEEE 33-Bus System with DG

Table 2. Network Reconstruction Switch Action Result

\begin{tabular}{cc}
\hline $\begin{array}{c}\text { Network } \\
\text { Condition }\end{array}$ & Opened Switches \\
\hline Excluding DG & $8-9,14-15,10-11,32-33,29-30$ \\
Including Two & $6-7,28-29,21-22,11-12,14-15,15-16,32-$ \\
DG & 33 \\
& \\
Including & $7-8,6-26,28-29,11-12,14-15,21-22,15-$ \\
Three DG & $16,32-33$ \\
\hline
\end{tabular}




\subsection{Taking IEEE69 Node System as an Example}

69 nodes, 74 branches, 5 interconnection switches, rated voltage at $12.66 \mathrm{kv}$, please refer to Diagram 7 and the specific system parameters are extracted from Document [7]. Install DG for grid-connected operation at Node 22 and 51. Crash happens to place between Branch 5 and 6.

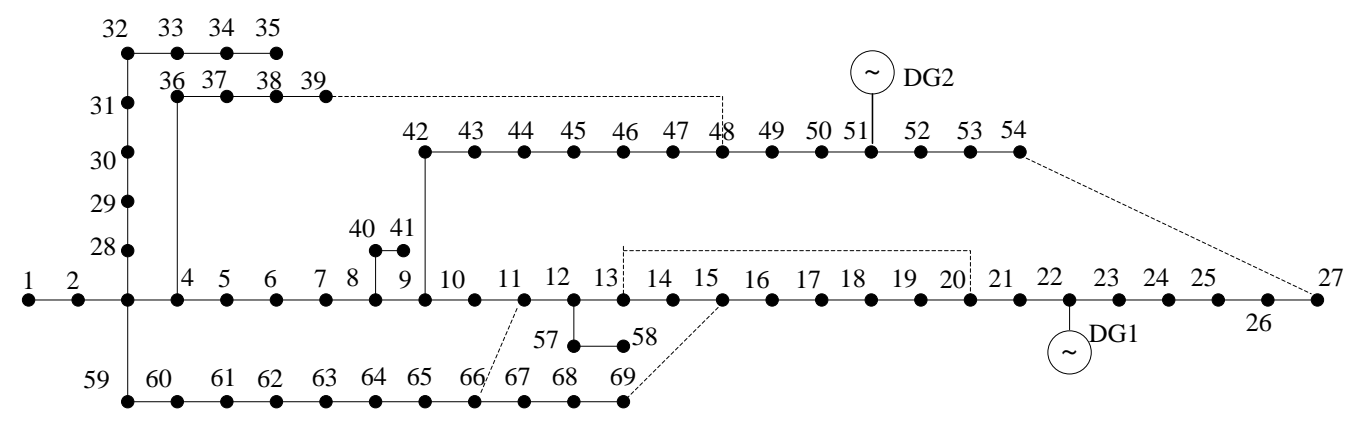

Figure 7. Diagram of leee69 Node System with One DG

When DG fails to conduct emergent power generation, conduct crash recovery switch action according to Table 3 and the power loss load after recovery is 0 . Network loss before crash is $324.46 \mathrm{~kW}$ and network loss after recovery is 140.9 $\mathrm{kW}$ and the network loss is reduced by using this method.

When DG is able to conduct emergent power generation and total DG capacity at $300 \mathrm{~kW}$, DG will form islanding power supply after crash and switch actions are as illustrated as those in Table 3. Total network loss including DG island is $117.65 \mathrm{kw}$.

Table 3. Network Reconstruction Switch Action Result

\begin{tabular}{cc}
\hline Network Condition & Opened Switches \\
\hline Excluding DG & $39-48,11-66,47-48$ \\
$\begin{array}{c}\text { DG Capacity at } \\
\text { 300kW }\end{array}$ & $18-19,39-48,11-66,47-48$ \\
\end{tabular}

\section{Conclusion}

In this paper, it puts forward a kind of power supply recovery algorithm for crash to distribution network with DG. Taking minimum network damage as its purpose, it uses depth search method to confirm islanding classification scheme; based on the improved branch change method, it judges the influence of switches' actions on network damage in order to simplify the crash recovery calculation process; as to the recovery of power loss area through reconstruction of distribution network, it uses islanding operation pattern to try to guarantee the continuous power supply by important loads. By using the heuristic method to conduct network power supply recovery and optimization to finally obtain the crash recovery scheme; finally, the author verifies the plausibility of this scheme through two numerical examples and it is able to effectively solve crash recovery problems for distribution network with DG and it contains certain value in projects. 


\section{Acknowledgements}

The project is sponsored by Shenyang Bureau of Science and Technology under Grant: F14-231-1-21. Liaoning Provincial Science and Technology under Grant: 2012201010, National Nature Science Foundation of China under Grant 61372195, and the Key Technologies R\&D Program of Liaoning Province under Grant 2012201010.

\section{References}

[1] C. S. Wang, "Analysis and Simulation Theory of Microgrids", Science Press, (2013).

[2] J. M. Guerrero, J. C. Vasquez and J. Matas, "Hierarchical Control of Droop-controlled AC and DC Microgrids-A General Approach toward Standardization”, IEEE Transactions on Industrial Electronics, vol. 58, no. 158, (2011).

[3] L. Jian, H. P. Dong and X. Y. Chen, "Fault Location and Restoration for Distribution Systems", Electric Power Press, (2012), Beijing China.

[4] X. YI and Y. P. LU, "Islanding Algorithm of Distribution Networks with Distributed Enerators", Power System Technology, vol. 30, no. 50, (2006).

[5] M. E. Baran and F. F. Wu, "Network reconfiguration in distribution systems for loss reduction and load balancing", IEEE Trans.on Power Delivery, vol. 4, no. 1401, (1989).

[6] L. Jian, P. X. Bi and H. P. Dong, "Simplification Analysis and Optimization of Complicated Distribution Network", Electric Power Press, (2002), Beijing, China.

\section{Authors}
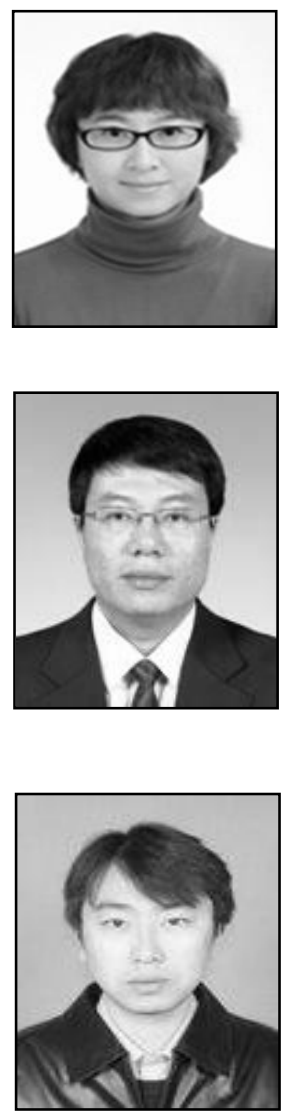

Di Bai, Associate Professor, Doctoral Student, Main study directions: distribution system analysis, distribution network crash recovery.

Tieyan Zhang, Professor, Doctoral Student Mentor, IEEE Member; Main study directions: grid combination technologies in intelligent power grid, diagnosis of complicated system faults, distributed power generation system modelling and analysis.

Zheng Yang, Associate Professor; main study direction: computer research and calculation. 
International Journal of Grid Distribution Computing

Vol. 8, No. 3, (2015) 